\title{
Factors affecting locoregional recurrence in breast cancer patients undergoing surgery following neoadjuvant treatment
}

Hsu-Huan Chou ${ }^{1,2^{*}}$, , Wei-Shan Chung ${ }^{1 \dagger}$, Rong-Yao Ding ${ }^{3}$, Wen-Ling Kuo ${ }^{1}$, Chi-Chang Yu ${ }^{1}$, Hsiu-Pei Tsai ${ }^{1}$, Shih-Che Shen', Chia-Hui Chu' ${ }^{1}$ Yung-Feng Lo ${ }^{1}$ and Shin-Cheh Chen ${ }^{1}$

\begin{abstract}
Background: Neoadjuvant chemotherapy (NAC) has been the standard treatment for locally advanced breast cancer for the purpose of downstaging or for conversion from mastectomy to breast conservation surgery (BCS). Locoregional recurrence (LRR) rate is still high after NAC. The aim of this study was to determine predictive factors for LRR in breast cancer patients in association with the operation types after NAC.
\end{abstract}

Methods: Between 2005 and 2017, 1047 breast cancer patients underwent BCS or mastectomy after NAC in Chang Gung Memorial Hospital, Linkou. We obtained data regarding patient and tumor characteristics, chemotherapy regimens, clinical tumor response, tumor subtypes and pathological complete response (pCR), type of surgery, and recurrence.

Results: The median follow-up time was 59.2 months (range 3.13-186.75 months). The mean initial tumor size was $4.89 \mathrm{~cm}(\mathrm{SD} \pm 2.95 \mathrm{~cm})$. Of the 1047 NAC patients, $232(22.2 \%)$ achieved pCR. The BCS and mastectomy rates were 41.3\% and 58.7\%, respectively. One hundred four patients developed LRR (9.9\%). Comparing between patients who underwent BCS and those who underwent mastectomy revealed no significant difference in the overall LRR rate of the two groups, $8.8 \%$ in BCS group vs $10.7 \%$ in mastectomy group $(p=0.303)$. Multivariate analysis indicated that independent factors for the prediction of LRR included clinical N2 status, negative estrogen receptor (ER), and failure to achieve pCR. In subgroups of multivariate analysis, only negative ER was the independent factor to predict LRR in mastectomy group ( $p=0.025$ ) and hormone receptor negative/human epidermal growth factor receptor 2 positive $(\mathrm{HR}-/ \mathrm{HER} 2+)$ subtype $(p=0.006)$ was an independent factor to predict LRR in BCS patients. Further investigation according to the molecular subtype showed that following BCS, non-pCR group had significantly increased LRR compared with the pCR group, in HR-/HER2 + subtype (25.0\% vs 8.3\%, $p=0.037)$, and HR-/HER2 - subtype (20.4\% vs $0 \%, p=0.002$ ).

Conclusion: Clinical N2 status, negative ER, and failure to achieve pCR after NAC were independently related to the risk of developing LRR. Operation type did not impact on the LRR. In addition, the LRR rate was higher in non-pCR hormone receptor-negative patients undergoing BCS comparing with pCR patients.

Keywords: Breast cancer, Neoadjuvant chemotherapy, Locoregional recurrence, Hormone receptor

*Correspondence: b9002009@cgmh.org.tw

${ }^{\dagger}$ Co-first author: Wei-Shan Chung

${ }^{1}$ Department of General Surgery, Chang Gung Memorial Hospital, Linkou,

No. 5, Fuxing St., Guishan Dist., Taoyuan City 333, Taiwan

Full list of author information is available at the end of the article

\section{Background}

Neoadjuvant chemotherapy (NAC) has generally been accepted as the standard treatment for locally advanced breast cancer. With the aim of making non-operable 
breast cancer operable, NAC increases the number of breast conservative therapy (BCS) candidates by downsizing locally advanced tumor [1, 2]. Hence, patients undergoing BCS after NAC might have a more favorable cosmetic outcome and quality of life [3].

Despite the fact that increased use of NAC has facilitated the treatment of breast cancer, long-term optimized outcomes and treatment effects are concerning. In their recently published meta-analysis on NAC and adjuvant chemotherapy, Asselain and colleagues reported that NAC has a higher local recurrence rate than adjuvant chemotherapy in patients with locally advanced tumors but no significant difference in terms of overall survival between patients undergoing NAC and adjuvant chemotherapy [4]. Similar results were reported in a meta-analysis by Mauri in 2005 [5]. The frequency of BCS, defined as lumpectomy, with whole breast irradiation, is higher after the use of NAC compared with adjuvant chemotherapy after surgery [4].

The long-term survival rate among early breast cancer women without NAC who undergo BCS was the same as that among women who undergo radical mastectomy $[6$, 7]. However, several studies have demonstrated no significant difference in long-term survival or local recurrence rates between women who underwent BCS and those who underwent radical mastectomy after $\operatorname{NAC}[8,9]$. Although predictive factors for local recurrence in these NAC-treated patient groups have not yet been clarified yet, the higher local recurrence risk of NAC than of adjuvant chemotherapy is controversial.

NAC is increasingly used in breast cancer therapy, and clinic-pathological subtypes, such as estrogen receptor positive (ER), progesterone receptor positive (PR), and human epidermal growth factor receptor 2 positive (HER-2), can provide prognostic information regarding the risk of local regional recurrence after NAC. Furthermore, pathological complete response (pCR) after NAC can significantly affect the prognosis of breast cancer $[10$, 11]. We would like to investigate these attributed factors for recurrence reported in retrospective studies on NAC. Furthermore, once the pCR-achieved status is confirmed, the omission of mastectomy is feasible. Patients receiving NAC, in total, have higher BCS rate in literature. We investigated these clinic-pathological attributed factors in association with the operation types after NAC.

\section{Methods}

The study was a retrospective cohort study and was approved by ethics committee of our institution and institutional review board number was 1711150042. Female patients diagnosed with histologically proven unilateral invasive breast cancer who received neo-adjuvant chemotherapy (NAC) and underwent mastectomy or breast conserving surgery at Chang Gung Memorial Hospital, Linkou, between 2005 and 2017 were enrolled. Exclusion criteria were inflammatory cancer, the initial presence of distant metastasis, synchronous bilateral breast cancer, loss of clinical information, and patients who did not undergo surgery after NAT either due to loss of follow up or due to progression development of metastasis after NAT. Clinical staging was determined through physical examination, mammography, ultrasonography of the breast and axillary lymph nodes, a bone scan, and a whole body computed tomography (CT) scan. Histological Diagnosis was confirmed by the specimen of core needle biopsy and abnormal axillary lymph node was routinely evaluated by fine needle aspiration. Clinical information including age, tumor histology, molecular subtype, TNM stage, Scarff-Bloom-Richardson (SBR) grade, NAC regimens, post-NAC response status, operation type, and locoregional recurrence were collected. Chemotherapy regimens included anthracycline-based and taxane-based regimens and the target therapy for HER2 contained trastuzumab and pertuzumab for some patients. Dose modifications were based on blood cell counts and adverse events. Preoperative radiological evaluation included breast ultrasonography and mammography. The range of resection would be decided as original tumor size while the scattered distribution of the tumor was observed after response of the treatment; the range of resection would be the new tumor size while concentric response after NAC according to the guideline of our institution. Operation including mastectomy or BCS and axillary sentinel lymph node biopsies or axillary lymph node dissections according to clinical posttreatment evaluation were performed 2-4 weeks after NAC was completed. The indication for postoperative radiotherapy included all patients after BCS and the patients receiving mastectomy with initial T3 stage (tumor size $>5 \mathrm{~cm}$ ) and initial N2 stage or residual positive lymph node after NAC. Almost all patients received postoperative adjuvant therapy, including hormone therapy, chemotherapy, and target therapy, according to their clinicopathological factors. Analysis of estrogen receptor, progesterone receptor, HER2 expression and the definition of $\mathrm{PCR}$ were described below. The analysis was performed using immunohistochemistry (IHC) staining techniques on pretreatment core needle biopsy specimens. For ER and PR, the positivity in $>1 \%$ of tumor cells was defined as expression. Hormone receptor (HR) positive was defined as ER or PR positive while HR negative was categorized as both negative ER and PR. The definition of positive HER2 was IHC staining with a score of $3+$ or a positive fluorescence in situ hybridization (FISH) test with IHC staining with a score of $2+$. $\mathrm{pCR}$ was defined as no residual invasive cancer cells in the breast and axillary lymph nodes (ypT0/ypTisN0). LRR 
was defined as ipsilateral breast tumor recurrence including invasive carcinoma and carcinoma in situ, chest wall or skin recurrence, and ipsilateral regional lymph node recurrence including the axilla, internal mammary and supraclavicular region.

\section{Statistical analysis}

Numerical data were compared using a Student's t-test and presented as the mean + standard deviation (SD) and a two-tailed $p$-value $<0.05$ was considered to be statistically significant. Pearson's chi-squared test for determining whether measurements from different groups are independent (the expected value in each cell is greater than 5), if an expected number is less than 5-> use Fisher's exact test of independence. To compare proportions of a categorical outcome according to different independent groups, we use the chi-squared test or Fisher's exact test when appropriate. A log-rank test and the KaplanMeier method were used for survival analysis. For multivariate analysis of prognostic factors, logistic regression was used. All analyses were performed using SPSS 22.0 for Windows (SPSS, Chicago, IL).

\section{Results}

During the study period, we enrolled 1047 consecutive patients with breast cancer who were treated with NAC followed by surgery and adjuvant therapy. The median follow-up time was 59.2 months (range 3.13-186.75 months) and the last follow up date was June 30, 2020. The mean initial tumor size was $4.89 \mathrm{~cm}$ $(\mathrm{SD} \pm 2.95 \mathrm{~cm})$. The T2 stage accounted for 582 patients (55.6\%), T3 for 205 patients (19.6\%), and T4 for 220 patients $(21.0 \%)$. For the clinical lymph node status, 79 patients $(7.5 \%)$ were N0, $543(51.9 \%)$ were N1, 425 (40.6\%) were N2. Overall, 1028 (98\%) were diagnosed with invasive ductal carcinoma, of whom 77 patients (7.3\%) had tumor SBR grade 1, 405 (38.7\%) had grade 2 for, and 466 (44.5\%) had grade 3 . According to IHC staining, $413(39.4 \%)$ patients were ER-negative, while 634 patients (60.6\%) were ER-positive; 520 (49.7\%) patients were PR-negative, while 527 (50.3\%) were PR-positive; and $466(44.5 \%)$ patients were HER-2 positive. The triple negative subtype (HR-/HER2-) accounted for $15.5 \%(\mathrm{n}=162)$ of patients, and the HR + /HER2 - subtype accounted for $40.0 \%(n=419)$. Regimens for NAC was prescribed according to the physician's preference. A total of 603 patients (57.7\%) were prescribed anthracycline-based agents combined with taxane-based regimens, while 325 patients (31.0\%) received chemotherapy agents combined with target therapies, of whom 68 patients received dual blockade therapy for HER2 + disease. Out of a total of 68 HER $2+$ patients receiving dual blockade therapy (trastuzumab + pertuzumab), 34 patients (50\%) achieved pCR while pCR occurred in 104 patients (40.5\%) out of 257 HER2 + patients with single blockade therapy $(p=0.1579)$. All patients underwent surgery after NAC; the BCS rate was $41.3 \%(n=432)$, and the mastectomy rate was $58.7 \%(\mathrm{n}=615)$. The overall $\mathrm{pCR}$ rate was $22.2 \%(\mathrm{n}=232)$, while the non-pCR rate was $77.8 \%(\mathrm{n}=815)$ (Table 1$)$.

Clinical T stage $(p<0.0001)$, clinical $\mathrm{N}$ stage $(p<0.0001)$, SBR grade $(p<0.0001)$, negative ER/PR $(p<0.0001)$, positive HER2 $(p<0.0001)$ and molecular subtype $(p<0.0001)$ were predictors of $\mathrm{pCR}$ in univariate analysis (Table 2). Multivariate analysis found similar results, including clinical T1 stage $(p<0.001)$ and clinical T2 $(p=0.028)$ as predictors of $\mathrm{pCR}$ compared with clinical T4 stage. Clinical N0 $(p=0.014)$ and clinical N1 $(p<0.0001)$ were also predictors of $\mathrm{pCR}$ compared with clinical N2 stage. SBR grade $3(p=0.033)$ was predictor of pCR compared with SBR grade 1. With regard to subtype classification, $\mathrm{HR}+/ \mathrm{HER} 2+(p<0.0001), \mathrm{HR}-1$ HER $2+(p<0.0001)$ and HR $-/$ HER $2-(p<0.0001)$ were predictors of pCR (Table 3 ).

During the follow up period, the recurrence rate was $26.8 \%(n=281)$, of which $9.9 \%(n=104)$ was locoregional recurrence (LRR) and $20.9 \%$ was distal metastasis. By the conclusion of this study, 191 (18.2\%) patients had died from breast cancer and 23 (2.2\%) patients had died from other causes. Furthermore, on univariate analysis of factors affecting LRR, we noted that clinical N2 stage $(p<0.0001)$, SBR grade $3(p=0.004)$, negative ER $(p=0.001)$, negative PR $(p=0.003)$, negative HR subtypes $(p=0.043)$, and non-pCR $(p=0.006)$ were predictors of LRR (Table 4). Comparing between patients who underwent BCS and those who underwent mastectomy revealed no significant difference in the overall LRR rate of the two groups, $8.8 \%$ in BCS group vs $10.7 \%$ in mastectomy group $(p=0.303)$. In multivariate analysis, only clinical N2 status $(p=0.018)$, negative ER $(p=0.011)$ and non-pCR $(p=0.019)$ were found to be predictors of LRR (Table 5). No significant difference of locoregional recurrence free survival rate (LRRFS) in the BCS and mastectomy groups are shown in Fig. $1(p=0.302)$. The effect of pCR on LRRFS is demonstrated in Fig. $2(p=0.004)$, as 5 -year LRRFS is $94.1 \%$ in PCR group and $87.9 \%$ in nonpCR group.

In patients who underwent mastectomy, clinical $\mathrm{T}$ stage $(p=0.019)$, clinical $\mathrm{N}$ stage $(p<0.001)$, negative ER $(p=0.032)$ and non-pCR $(p=0.034)$ were factors to predict LRR in univariate analysis. In multivariate analysis, only negative ER remained an independent factor for unfavorable LRR compared with ER positive patients in mastectomy group $(p=0.025)$ (Table 6). The LRRFS rate in the $\mathrm{PCR}$ group following mastectomy showed a favorable outcome $(p=0.034)$, as shown in Fig. 3, while 
Table 1 Clinicopathological characteristics of the study population

\begin{tabular}{|c|c|}
\hline Parameters & No. of cases \\
\hline Age (years), median (IQR) & $49(14)$ \\
\hline Tumor size (cm), median (IQR) & $4.1(2.3)$ \\
\hline \multicolumn{2}{|l|}{ Clinical T stage } \\
\hline $\mathrm{T} 1$ & $40(3.8 \%)$ \\
\hline $\mathrm{T} 2$ & $582(55.6 \%)$ \\
\hline T3 & $205(19.6 \%)$ \\
\hline $\mathrm{T} 4$ & $220(21.0 \%)$ \\
\hline \multicolumn{2}{|l|}{ Clinical lymph node status } \\
\hline No & $79(7.5 \%)$ \\
\hline N1 & $543(51.9 \%)$ \\
\hline N2 & $425(40.6 \%)$ \\
\hline \multicolumn{2}{|l|}{ Histologic type } \\
\hline Invasive ductal carcinoma & $1028(98.1 \%)$ \\
\hline Mucinous carcinoma & $4(0.4 \%)$ \\
\hline Invasive lobular carcinoma & $10(1.0 \%)$ \\
\hline Invasive micropapillary carcinoma & $5(0.5 \%)$ \\
\hline \multicolumn{2}{|l|}{ SBR grade } \\
\hline 1 & $77(7.3 \%)$ \\
\hline 2 & $405(38.7 \%)$ \\
\hline 3 & $466(44.5 \%)$ \\
\hline Unknown & $99(9.5 \%)$ \\
\hline \multicolumn{2}{|l|}{ ER status } \\
\hline Negative & $413(39.4 \%)$ \\
\hline Positive & $634(60.6 \%)$ \\
\hline \multicolumn{2}{|l|}{ PR status } \\
\hline Negative & $520(49.7 \%)$ \\
\hline Positive & $527(50.3 \%)$ \\
\hline \multicolumn{2}{|l|}{ HER2 status } \\
\hline Negative & $581(55.5 \%)$ \\
\hline Positive & $466(44.5 \%)$ \\
\hline \multicolumn{2}{|l|}{ Subtype } \\
\hline $\mathrm{HR}+/ \mathrm{HER} 2-$ & $419(40.0 \%)$ \\
\hline $\mathrm{HR}+/ \mathrm{HER} 2+$ & $237(22.6 \%)$ \\
\hline HR-/HER2 + & $229(21.9 \%)$ \\
\hline HR-/HER2- & $162(15.5 \%)$ \\
\hline \multicolumn{2}{|l|}{ Surgery } \\
\hline Mastectomy & $615(58.7 \%)$ \\
\hline Breast conserving surgery & $432(41.3 \%)$ \\
\hline \multicolumn{2}{|l|}{ Margin } \\
\hline Free & $1014(96.8 \%)$ \\
\hline Positive & $33(3.2 \%)$ \\
\hline \multicolumn{2}{|l|}{ Neoadjuvant regimens } \\
\hline Anthracycline only & $37(3.5 \%)$ \\
\hline Taxane only & $82(7.8 \%)$ \\
\hline Antracycline + taxane & $603(57.7 \%)$ \\
\hline Chemotherapy + target therapy & $325(31.0 \%)$ \\
\hline \multicolumn{2}{|l|}{ Pathological response } \\
\hline $\mathrm{pCR}$ & $232(22.2 \%)$ \\
\hline Non-pCR & $815(77.8 \%)$ \\
\hline
\end{tabular}

Table 1 (continued)

ER estrogen receptor, $H R$ hormone receptor, $H E R$-2 human epidermal growth factor receptor $2, p C R$ pathological complete response, $P R$ progesterone receptor

Table 2 Comparison between patients pCR and non-pCR

\begin{tabular}{|c|c|c|c|}
\hline Parameters & $\begin{array}{l}\mathrm{pCR} \\
(\mathrm{n}=232)\end{array}$ & $\begin{array}{l}\text { non-pCR } \\
(n=815)\end{array}$ & P value \\
\hline Age (years) & & & 0.493 \\
\hline$\leqq 50$ & $132(23.0 \%)$ & 443 (77.0\%) & \\
\hline$>50$ & $100(21.2 \%)$ & $372(78.8 \%)$ & \\
\hline Clinical T stage & & & $<0.0001$ \\
\hline $\mathrm{T} 1$ & $17(42.5 \%)$ & $23(57.5 \%)$ & \\
\hline $\mathrm{T} 2$ & $149(25.6 \%)$ & $433(74.4 \%)$ & \\
\hline T3 & $39(19.0 \%)$ & $166(81.0 \%)$ & \\
\hline $\mathrm{T} 4$ & $27(12.3 \%)$ & $193(87.7 \%)$ & \\
\hline Clinical lymph node status & & & $<0.0001$ \\
\hline NO & $19(24.1 \%)$ & $60(75.9 \%)$ & \\
\hline N1 & $164(30.2 \%)$ & $379(69.8 \%)$ & \\
\hline N2 & $49(11.5 \%)$ & $376(88.5 \%)$ & \\
\hline SBR grade & & & $<0.0001$ \\
\hline 1 & $4(5.2 \%)$ & $73(94.8 \%)$ & \\
\hline 2 & $51(12.6 \%)$ & $354(87.4 \%)$ & \\
\hline 3 & $110(23.6 \%)$ & $356(76.4 \%)$ & \\
\hline Unknown & $67(67.7 \%)$ & $32(32.3 \%)$ & \\
\hline ER status & & & $<0.0001$ \\
\hline Negative & $144(32.9 \%)$ & $269(65.1 \%)$ & \\
\hline Positive & $88(13.9 \%)$ & $546(86.1 \%)$ & \\
\hline PR status & & & $<0.0001$ \\
\hline Negative & $172(33.1 \%)$ & $348(66.9 \%)$ & \\
\hline Positive & $60(11.4 \%)$ & $467(88.6 \%)$ & \\
\hline HER2 status & & & $<0.0001$ \\
\hline Negative & $78(13.4 \%)$ & $503(86.6 \%)$ & \\
\hline Positive & $154(33.0 \%)$ & $312(67.0 \%)$ & \\
\hline Subtype & & & $<0.0001$ \\
\hline $\mathrm{HR}+/ \mathrm{HER} 2-$ & $27(6.4 \%)$ & $392(93.6 \%)$ & \\
\hline $\mathrm{HR}+/ \mathrm{HER} 2+$ & $66(27.8 \%)$ & $171(72.2 \%)$ & \\
\hline $\mathrm{HR}-/ \mathrm{HER} 2+$ & 88 (38.4\%) & $141(61.6 \%)$ & \\
\hline HR-/HER2- & $51(31.5 \%)$ & 111 (68.5\%) & \\
\hline
\end{tabular}

$E R$ estrogen receptor, $H R$ hormone receptor, $H E R-2$ human epidermal growth factor receptor $2, p C R$ pathological complete response

5-year LRRFS was $92.4 \%$ in pCR group and $88.2 \%$ in non-pCR group. In BCS group, negative ER $(p=0.005)$, negative PR (0.004) and negative HR molecular subtype $(p=0.029)$ were predictors to LRR in univariate analysis. HR-/HER2 + subtype $(p=0.006)$ was an independent factor to predict LRR in BCS patients (Table 7). LRRFS in BCS group was also found to be numerically, but not significant, different between the pCR and non-pCR groups, as shown in Fig. $4(p=0.080)$. Further analysis focusing 
Table 3 Multivariate analysis of factors predicting pCR after NAC

\begin{tabular}{|c|c|c|c|}
\hline Parameters & Odds ratio & 95\% Confidence interval & $P$ value \\
\hline \multicolumn{4}{|l|}{ Clinical T stage } \\
\hline T1 vs T4 & 5.304 & $2.222-12.664$ & $<0.001$ \\
\hline T2 vs T4 & 1.808 & $1.065-3.067$ & 0.028 \\
\hline T3 vs T4 & 1.216 & $0.648-2.282$ & 0.541 \\
\hline \multicolumn{4}{|l|}{ Clinical N stage } \\
\hline No vs N2 & 2.434 & $1.201-4.933$ & 0.014 \\
\hline N1 vs N2 & 3.344 & $2.196-5.090$ & $<0.0001$ \\
\hline \multicolumn{4}{|l|}{ SBR grade } \\
\hline $2 / 1$ & 2.327 & $0.765-7.073$ & 0.137 \\
\hline $3 / 1$ & 3.312 & $1.101-9.963$ & 0.033 \\
\hline \multicolumn{4}{|l|}{ Subtype } \\
\hline $\mathrm{HR}+/ \mathrm{HER} 2+\mathrm{vs} \mathrm{HR}+/ \mathrm{HER} 2-$ & 4.851 & $2.862-8.221$ & $<0.0001$ \\
\hline $\mathrm{HR}-/ \mathrm{HER} 2+\mathrm{vs} \mathrm{HR}+/ \mathrm{HER} 2-$ & 8.781 & $5.146-14.986$ & $<0.0001$ \\
\hline HR -/HER2 - vs HR + /HER2- & 4.868 & $2.722-8.706$ & $<0.0001$ \\
\hline
\end{tabular}

$H R$ hormone receptor, HER-2 human epidermal growth factor receptor 2, NAC neoadjuvant chemotherapy, $p C R$ pathological complete response

on the subtypes of the BCS group demonstrated that the HR-/HER2 + non-pCR group had significantly increased LRR compared with the HR-/HER2 + pCR group $(25.0 \%$ vs $8.3 \%, p=0.037)$. Furthermore, the HR-/HER2non-pCR group had significantly increased LRR compared with the HR-/HER2-pCR group (20.4\% vs $0 \%$, $p=0.002$ ). (Table 8 ). Moreover, there was no difference in LRR found with regards to hormone receptor positive disease between the between pCR and non-pCR groups. Further analysis of molecular subtypes indicated no significant difference for all subtypes between the pCR and non-pCR groups following mastectomy (Table 8).

\section{Discussion}

In total, 232 patients (22.2\%) achieved pCR. Multivariate analysis indicated that clinical $\mathrm{T}$ stage, clinical $\mathrm{N}$ stage, and molecular subtype were independent predictors to pCR. A total of 104 patients $(9.9 \%)$ developed LRR of which 12 were in the pCR group and 92 were in the nonpCR group. Our study reported that pCR in all breast cancer subtypes, after NAC, provided better local control. The result was in line with the findings of our previous report published in 2018, in which no LRR occurred in the $\mathrm{PCR}$ group and 31 patients (13.2\%) in the non-pCR group with significant difference in total 263 patients all receiving neoadjuvant weekly epirubicin and docetaxel regimens[12]. Our study revealed that 232 patients (22.2\%) achieved pCR among the 1047 patients underwent while the BCS rate is $41 \%$ and the rest of patients received mastectomy (59\%). Overall, 281 patients experienced tumor recurrence (26.8\%).

Although the pooled analysis from CTNeoBC did not support $\mathrm{pCR}$ as a surrogate endpoint for an improved event-free survival or overall survival in all subtypes of breast cancer [13], pCR was an effective surrogate endpoint for selected patients in aggressive subtypes including luminal B/HER-, HER2 overexpression and triple negative breast cancer [14]. A recent comprehensive meta-analysis concluded that $\mathrm{pCR}$ followed by NAC was associated with significantly better event-free survival (EFS) and overall survival (OS), especially for patients with triple-negative and HER2 + breast cancer. The tumor response effect observed in the pCR group was similar in adjuvant chemotherapy and NAC patients [15]. Moreover, data from the combined analysis of the National Surgical Adjuvant Breast and Bowel Project (NSABP) B-18 and B-27 showed that the residual tumor status was an independent predictor of LRR in all patients at the 10 year follow-up, regardless of surgery type [10]. Another large analysis of the European Organisation for Research and Treatment of Cancer (EORTC) 10,994/BIG 1-00 study of patients with locally advanced breast cancer receiving NAC showed that $\mathrm{PCR}$ was a favorable factor with regards to the prediction of LRR after NAC [16]. Several retrospective series also demonstrated that achieving $\mathrm{pCR}$ after NAC can result in better local control following surgery [1, $2,17,18]$. Therefore, achieving pCR was an important factor not only for distant disease control but also for local control. In our series, failure to achieve pCR was also proved to be an independent predictor of LRR in total population. In subgroup analysis of mastectomy patients, the effect of pCR was only demonstrated in univariate analysis while numerically but not significantly lower LRR in pCR group comparing non-pCR group in patients undergoing BCS. 
Table 4 Factors predicting LRR after NAC

\begin{tabular}{|c|c|c|c|}
\hline Parameters & $\begin{array}{l}\text { LRR } \\
(n=104)\end{array}$ & $\begin{array}{l}\text { No LRR } \\
(n=943)\end{array}$ & Pvalue \\
\hline Age (years) & & & 0.66 \\
\hline$\leqq 50$ & $55(9.6 \%)$ & $520(90.4 \%)$ & \\
\hline$>50$ & $49(10.4 \%)$ & $423(89.6 \%)$ & \\
\hline Clinical T stage & & & 0.059 \\
\hline $\mathrm{T} 1$ & $5(12.5 \%)$ & 35 (87.5\%) & \\
\hline $\mathrm{T} 2$ & $51(8.8 \%)$ & $531(91.2 \%)$ & \\
\hline T3 & $16(7.8 \%)$ & $189(92.2 \%)$ & \\
\hline $\mathrm{T} 4$ & $32(14.5 \%)$ & $188(85.5 \%)$ & \\
\hline Clinical lymph node status & & & $<0.0001$ \\
\hline No & $2(2.5 \%)$ & 77 (97.5\%) & \\
\hline N1 & $40(7.4 \%)$ & $503(92.6 \%)$ & \\
\hline N2 & $62(14.6 \%)$ & $363(85.4 \%)$ & \\
\hline SBR grade & & & 0.004 \\
\hline 1 & $2(2.6 \%)$ & $75(97.4 \%)$ & \\
\hline 2 & $38(9.4 \%)$ & 367 (90.6\%) & \\
\hline 3 & 60 (12.9\%) & $406(87.1 \%)$ & \\
\hline Unknown & $4(4.0 \%)$ & $95(96.0 \%)$ & \\
\hline Histologic type & & & 0.083 \\
\hline Invasive ductal carcinoma & $100(9.7 \%)$ & $928(90.3 \%)$ & \\
\hline Invasive lobular carcinoma & $2(20.0 \%)$ & $8(80.0 \%)$ & \\
\hline Mucinous carcinoma & 0 & $4(100.0 \%)$ & \\
\hline Others & $2(40.0 \%)$ & $3(60.0 \%)$ & \\
\hline Margin & & & 0.765 \\
\hline Free & $102(10.1 \%)$ & 912 (89.9\%) & \\
\hline Positive & $2(6.1 \%)$ & $31(93.9 \%)$ & \\
\hline ER & & & 0.001 \\
\hline Positive & $47(7.4 \%)$ & $587(92.6 \%)$ & \\
\hline Negative & $57(13.8 \%)$ & $356(86.2 \%)$ & \\
\hline PR & & & 0.003 \\
\hline Positive & $38(7.2 \%)$ & $489(92.8 \%)$ & \\
\hline Negative & $66(12.7 \%)$ & $454(87.3 \%)$ & \\
\hline HER2 & & & 0.722 \\
\hline Positive & $48(10.3 \%)$ & $418(89.7 \%)$ & \\
\hline Negative & $56(9.6 \%)$ & $525(90.4 \%)$ & \\
\hline Subtype & & & 0.043 \\
\hline $\mathrm{HR}+/ \mathrm{HER} 2-$ & $33(7.9 \%)$ & $386(92.1 \%)$ & \\
\hline $\mathrm{HR}+/ \mathrm{HER} 2+$ & $19(8.0 \%)$ & $218(92.0 \%)$ & \\
\hline HR-/HER2 + & $29(12.7 \%)$ & $200(87.3 \%)$ & \\
\hline HR-/HER2- & $23(14.2 \%)$ & $139(85.8 \%)$ & \\
\hline Neoadjuvant regimens & & & 0.189 \\
\hline Anthracycline only & $7(18.9 \%)$ & $30(81.1 \%)$ & \\
\hline Taxane only & $6(7.3 \%)$ & $76(92.7 \%)$ & \\
\hline Anthracycline + taxane & $63(10.4 \%)$ & $540(89.6 \%)$ & \\
\hline Chemotherapy + target therapy & $28(8.6 \%)$ & $297(91.4 \%)$ & \\
\hline Operation type & & & 0.303 \\
\hline Mastectomy & $66(10.7 \%)$ & $549(89.3 \%)$ & \\
\hline $\mathrm{BCS}$ & $38(8.8 \%)$ & $394(91.2 \%)$ & \\
\hline $\mathrm{pCR}$ & & & 0.006 \\
\hline Yes & $12(5.2 \%)$ & $220(94.8 \%)$ & \\
\hline No & $92(11.3 \%)$ & $723(88.7 \%)$ & \\
\hline
\end{tabular}

Table 4 (continued)

ER estrogen receptor, $H R$ hormone receptor, $H E R-2$ human epidermal growth factor receptor 2, LRR locoregional recurrence, NAC neoadjuvant chemotherapy, $p C R$ pathological complete response, $P R$ progesterone receptor

Table 5 Multivariate analysis of factors to predict LRR

\begin{tabular}{|c|c|c|c|}
\hline Parameters & Odds ratio & 95\% Confidence interval & $P$ value \\
\hline \multicolumn{4}{|c|}{$\begin{array}{l}\text { Clinical lymph node } \\
\text { status }\end{array}$} \\
\hline No & 1 & & \\
\hline N1 & 2.981 & $0.700-12.701$ & 0.14 \\
\hline N2 & 5.668 & $1.343-23.929$ & 0.018 \\
\hline \multicolumn{4}{|l|}{ SBR grade } \\
\hline 1 & 1 & & \\
\hline 2 & 3.012 & $0.700-12.954$ & 0.139 \\
\hline 3 & 3.572 & $0.827-15.428$ & 0.088 \\
\hline Unknown & 1.541 & $0.259-9.157$ & 0.634 \\
\hline \multicolumn{4}{|l|}{ ER } \\
\hline Positive & 1 & & \\
\hline Negative & 4.272 & $1.405-12.995$ & 0.011 \\
\hline \multicolumn{4}{|l|}{ PR } \\
\hline Positive & 1 & & \\
\hline Negative & 1.768 & $0.896-3.487$ & 0.1 \\
\hline \multicolumn{4}{|l|}{ Subtype } \\
\hline $\mathrm{HR}+/ \mathrm{HER} 2-$ & 1 & & \\
\hline $\mathrm{HR}+/ \mathrm{HER} 2+$ & 1.029 & $0.552-1.919$ & 0.929 \\
\hline HR-/HER2 + & 0.295 & $0.075-1.159$ & 0.08 \\
\hline HR-/HER2- & 0.329 & $0.083-1.313$ & 0.115 \\
\hline \multicolumn{4}{|l|}{$\mathrm{pCR}$} \\
\hline Yes & 1 & & \\
\hline No & 2.247 & $1.146-4.407$ & 0.019 \\
\hline
\end{tabular}

$H R$ hormone receptor, HER-2 human epidermal growth factor receptor 2, LRR locoregional recurrence, $p C R$ pathological complete response

The results from the EORTC 10994/BIG 1-00 study of patients with locally advanced breast cancer receiving NAC showed that breast cancer subtypes, including HER2 + with or without trastuzumab and triple-negative, are predictive factors for high LRR after NAC [16]. Yang et al. reported that 233 stage II-III breast cancer patients treated with NAC, mastectomy, and post-mastectomy radiotherapy had an $8 \%$ LRR rate over 5 years with a 62-month median follow-up. The authors concluded that patients with triple-negative breast cancer had the highest LRR rate and those with HR + and HER2 + breast cancer had favorable LRR rates, regardless of NAC response [18]. In other several retrospective studies, molecular subtypes including HER2 + and triple-negative also showed poor LRR in BCS patients $[1,2]$. In our study, negative ER was found an independent significant factor for the prediction of LRR, regardless of treatment response. Negative ER remained an independent factor 


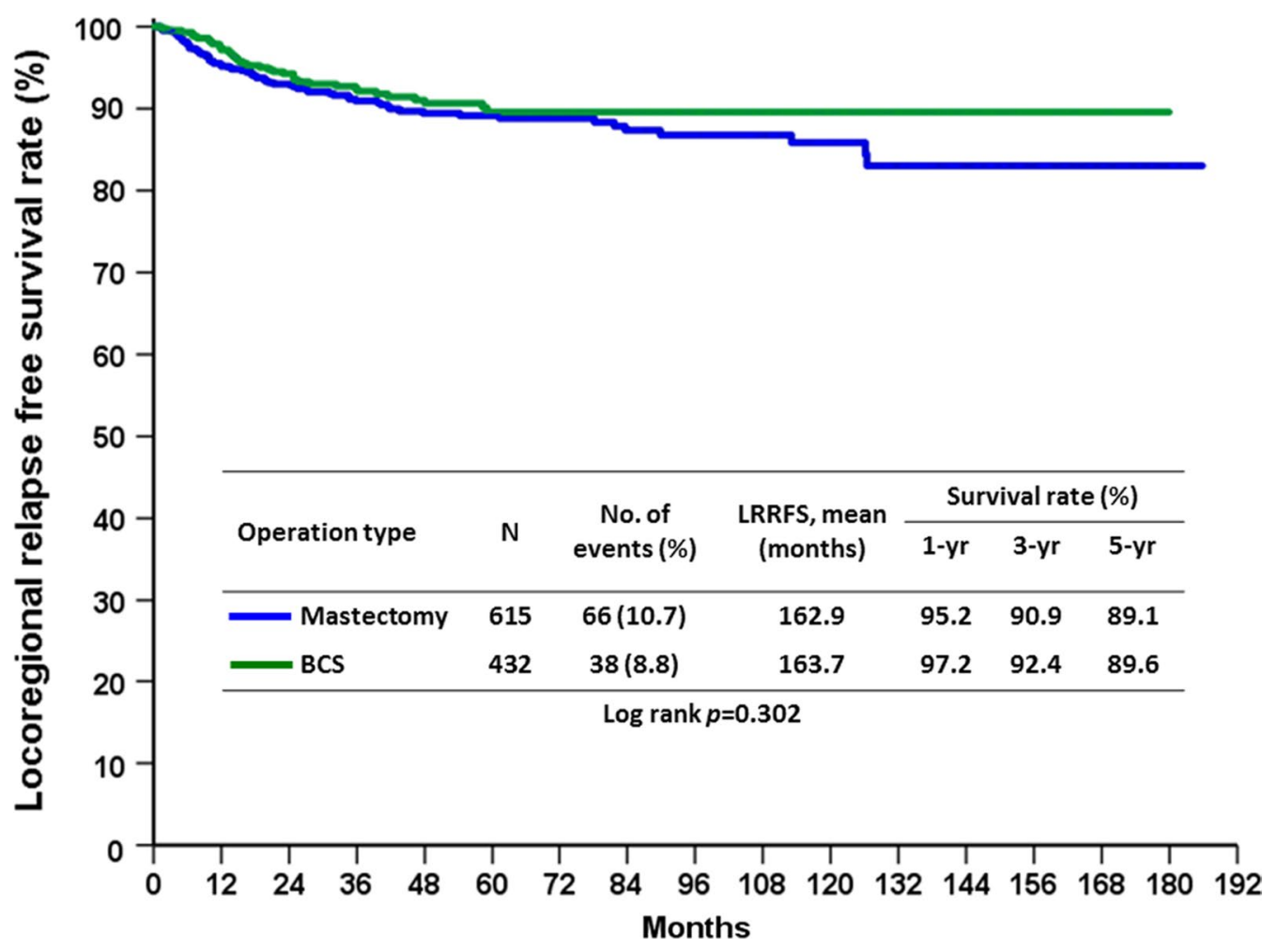

Fig. 1 LRRFS rate in the BCS group compared with the mastectomy group

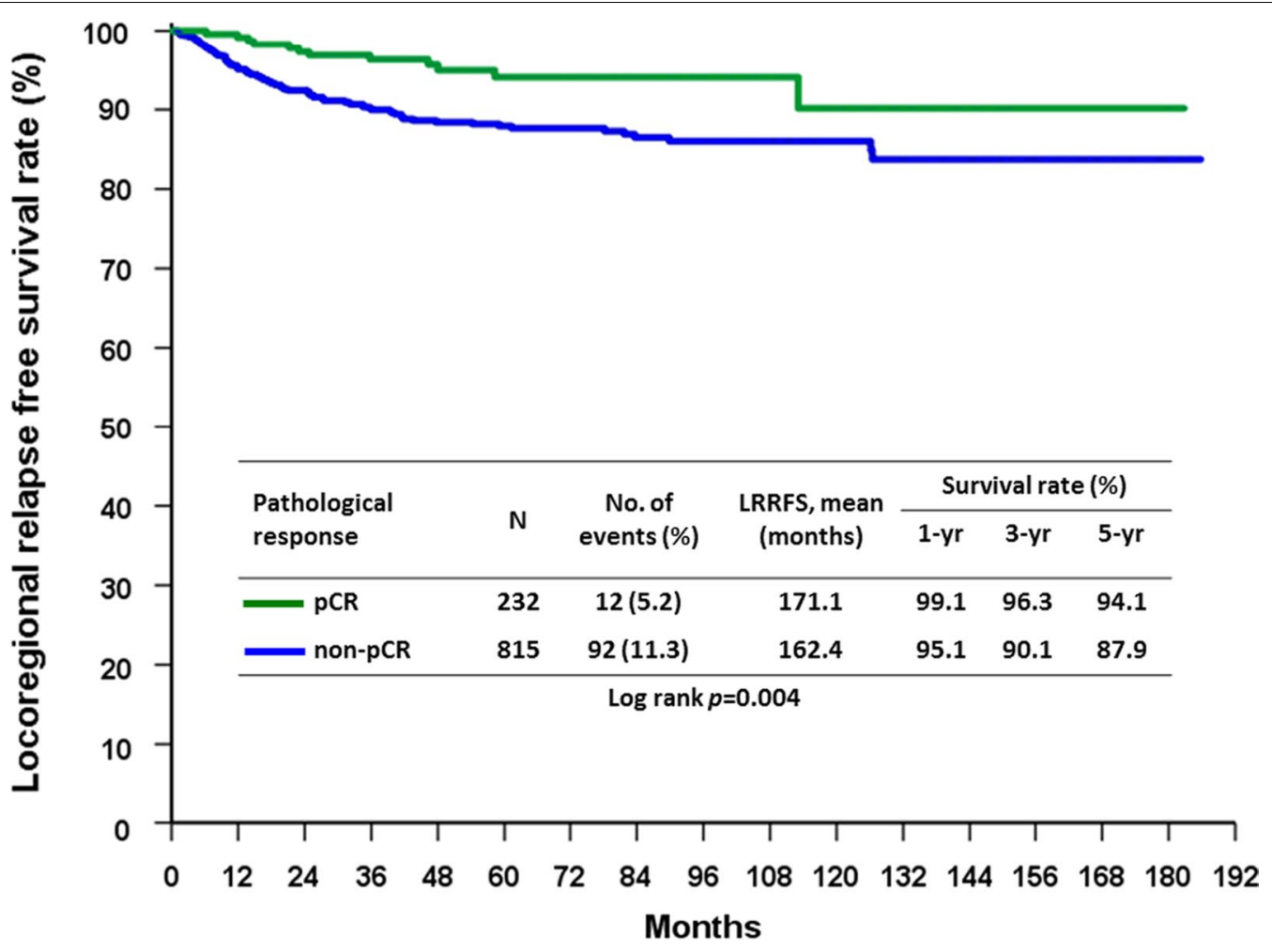

Fig. 2 LRRFS rate in the $p C R$ group compared with the non-pCR group 
Table 6 Univariate and multivariate analysis of factors predicting LRR in patients undergoing mastectomy after NAC

\begin{tabular}{|c|c|c|c|c|c|c|}
\hline \multirow[t]{2}{*}{ Parameters } & \multicolumn{3}{|c|}{ Univariate analysis } & \multicolumn{3}{|c|}{ Multivariate analysis } \\
\hline & $\operatorname{LRR}(n=66)$ & No LRR $(n=549)$ & $P$ value & Odds ratio & 95\% Confidence interval & $P$ value \\
\hline Age (years) & & & 0.477 & & & \\
\hline$\leqq 50$ & $30(9.8 \%)$ & $275(90.2 \%)$ & & & & \\
\hline$>50$ & $36(11.6 \%)$ & $274(88.4 \%)$ & & & & \\
\hline Clinical T stage & & & 0.019 & & & \\
\hline $\mathrm{T} 1$ & $3(20.0 \%)$ & $12(80.0 \%)$ & & 1 & & \\
\hline $\mathrm{T} 2$ & $23(9.1 \%)$ & $229(90.9 \%)$ & & 0.335 & $0.081-1.380$ & 0.13 \\
\hline T3 & $9(6.1 \%)$ & $138(93.9 \%)$ & & 0.228 & $0.050-1.035$ & 0.055 \\
\hline T4 & $31(15.4 \%)$ & $170(84.6 \%)$ & & 0.434 & $0.105-1.782$ & 0.246 \\
\hline Clinical lymph node status & & & $<0.001$ & & & \\
\hline NO & $1(4.8 \%)$ & $20(95.2 \%)$ & & 1 & & \\
\hline N1 & $16(5.7 \%)$ & $264(94.3 \%)$ & & 1.223 & $0.151-9.914$ & 0.85 \\
\hline N2 & $49(15.6 \%)$ & $265(84.4 \%)$ & & 2.983 & $0.380-23.421$ & 0.299 \\
\hline SBR grade & & & 0.07 & & & \\
\hline 1 & $1(2.1 \%)$ & $46(97.9 \%)$ & & & & \\
\hline 2 & $25(10.3 \%)$ & $217(89.7 \%)$ & & & & \\
\hline 3 & $37(13.5 \%)$ & $238(86.5 \%)$ & & & & \\
\hline Unknown & $3(5.9 \%)$ & $48(94.1 \%)$ & & & & \\
\hline Histologic type & & & 0.534 & & & \\
\hline Invasive ductal carcinoma & $64(10.6 \%)$ & $539(89.4 \%)$ & & & & \\
\hline Invasive lobular carcinoma & $1(16.7 \%)$ & $5(83.3 \%)$ & & & & \\
\hline Mucinous carcinoma & 0 & $3(100.0 \%)$ & & & & \\
\hline Others & $1(33.3 \%)$ & $2(66.7 \%)$ & & & & \\
\hline Margin & & & $>0.999$ & & & \\
\hline Free & $66(10.1 \%)$ & $541(89.1 \%)$ & & & & \\
\hline Positive & 0 & $8(100.0 \%)$ & & & & \\
\hline ER & & & 0.032 & & & \\
\hline Positive & $33(8.6 \%)$ & $349(91.4 \%)$ & & 1 & & \\
\hline Negative & $33(14.2 \%)$ & $200(85.8 \%)$ & & 1.837 & $1.078-3.131$ & 0.025 \\
\hline PR & & & 0.105 & & & \\
\hline Positive & $29(8.8 \%)$ & $299(91.2 \%)$ & & & & \\
\hline Negative & $37(12.9 \%)$ & $250(87.1 \%)$ & & & & \\
\hline HER2 & & & 0.51 & & & \\
\hline Positive & $27(9.8 \%)$ & $248(90.2 \%)$ & & & & \\
\hline Negative & $39(11.5 \%)$ & $301(88.5 \%)$ & & & & \\
\hline Subtype & & & 0.206 & & & \\
\hline HR + /HER2- & $26(9.8 \%)$ & $240(90.2 \%)$ & & & & \\
\hline $\mathrm{HR}+/ \mathrm{HER} 2+$ & $11(8.5 \%)$ & $119(91.5 \%)$ & & & & \\
\hline HR-/HER2 + & $16(11.0 \%)$ & $129(89.0 \%)$ & & & & \\
\hline HR-/HER2- & $13(17.6 \%)$ & $61(82.4 \%)$ & & & & \\
\hline Neoadjuvant regimens & & & 0.229 & & & \\
\hline Anthracycline only & $5(19.2 \%)$ & $21(80.8 \%)$ & & & & \\
\hline Taxane only & $5(11.4 \%)$ & $39(88.6 \%)$ & & & & \\
\hline Anthracycline + Taxane & $44(11.6 \%)$ & $336(88.4 \%)$ & & & & \\
\hline Chemotherapy + Target therapy & $12(7.3 \%)$ & $153(92.7 \%)$ & & & & \\
\hline $\mathrm{pCR}$ & & & 0.034 & & & \\
\hline Yes & $4(4.4 \%)$ & 87 (95.6\%) & & 1 & & \\
\hline No & $62(11.8 \%)$ & $462(88.2 \%)$ & & 2.524 & $0.856-7.444$ & 0.093 \\
\hline
\end{tabular}




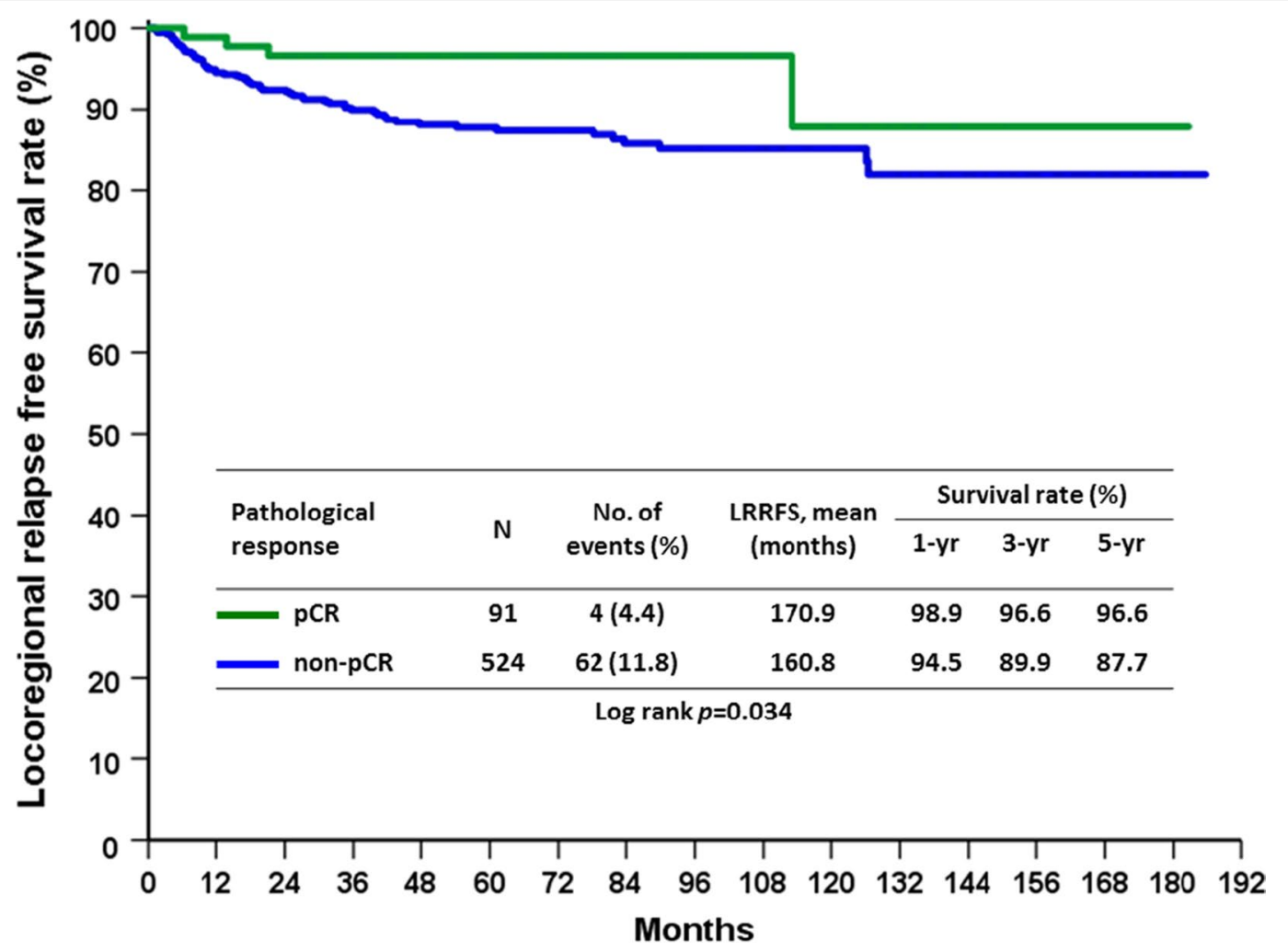

Fig. 3 LRRFS rate in the $p C R$ group compared with the non-pCR group in patients who underwent mastectomy

for unfavorable LRR compared with ER positive patients in mastectomy group and HR-/HER2 + subtype was an independent factor to predict LRR in BCS patients. The status of the hormone receptor appears to play a more important role in LRR than the NAC treatment response in our results.

The Early Breast Cancer Trialists' Collaborative Group (EBCTCG) recently reported that NAC was associated with more frequent local recurrence than that of adjuvant chemotherapy. The 15-year local recurrence rate was reported to be $21.4 \%$ for NACT compared with $15.9 \%$ for adjuvant chemotherapy from a meta-analysis of individual patient data from 10 randomized trials with average 9 years of follow-up. The study group also found that patients who underwent NAC had an increased frequency of breast-conserving therapy (65\%) versus those treated with adjuvant chemotherapy (49\%). The largest difference of LRR appeared in planned mastectomy patients and surgery less commonly used patients. The authors concluded tumors downsized by NAC might have higher local recurrence after BCS than tumors of the same dimensions in women who did not undergo NAC. Furthermore, the majority of patients only received anthracycline-based chemotherapy and final enrollment of patients occurred in 2002 [4]. Previous NSABP B-27 trial reports indicated that anthracycline-based regimens with the addition of taxane were associated with higher pCR rates and better local control [19]. Moreover, neoadjuvant chemotherapy plus trastuzumab was shown to be a predictor factor for favorable long-term survival but trastuzumab cannot be used before 2002 [20]. In our studies, the majority of patients received anthracycline-based regimens combined with taxane-based chemotherapy and every patient underwent appropriate surgery. Results from the combined study of NSABP B-18 and B-27 revealed that the 10-year LRR was $12.3 \%$ for patients with mastectomy and $10.3 \%$ for patients with lumpectomy plus whole breast radiotherapy, indicating no significant difference between the mastectomy and $\mathrm{BCS}$ groups following NAC [10]. In the I-SPY trial there was no substantial difference in LRR between the mastectomy and BCS groups, given the fact that the mastectomy group on average had higher clinical staging [21]. A higher breast conservation rate would not increase the LRR rate, which the NSABP B-18 and the EORTC studies have confirmed [22, 23]. In another pooled analysis of 5500 participants, the mastectomy rate in NAC group was found to be lower than in the adjuvant chemotherapy group, without interfering the local control [24]. In our report, 615 (58.7\%) patients chose mastectomy, while 432 
Table 7 Univariate and multivariate analysis of factors predicting LRR in patients undergoing BCS after NAC

\begin{tabular}{|c|c|c|c|c|c|c|}
\hline \multirow[t]{2}{*}{ Parameters } & \multicolumn{3}{|c|}{ Univariate analysis } & \multicolumn{3}{|c|}{ Multivariate analysis } \\
\hline & $\operatorname{LRR}(n=38)$ & No LRR $(n=394)$ & $P$ value & Odds ratio & 95\% confidence interval & $P$ value \\
\hline Age (years) & & & 0.661 & & & \\
\hline$\leqq 50$ & $25(9.3 \%)$ & $245(90.7 \%)$ & & & & \\
\hline$>50$ & $13(8.0 \%)$ & $149(92.0 \%)$ & & & & \\
\hline Clinical T stage & & & 0.77 & & & \\
\hline $\mathrm{T} 1$ & $2(8.0 \%)$ & $23(92.0 \%)$ & & & & \\
\hline $\mathrm{T} 2$ & $28(8.5 \%)$ & $302(91.5 \%)$ & & & & \\
\hline T3 & $7(12.1 \%)$ & $51(87.9 \%)$ & & & & \\
\hline $\mathrm{T} 4$ & $1(5.3 \%)$ & $18(94.7 \%)$ & & & & \\
\hline Clinical lymph node status & & & 0.089 & & & \\
\hline NO & $1(1.7 \%)$ & $57(98.3 \%)$ & & & & \\
\hline $\mathrm{N} 1$ & $24(9.1 \%)$ & $239(90.9 \%)$ & & & & \\
\hline $\mathrm{N} 2$ & $13(11.7 \%)$ & $98(88.3 \%)$ & & & & \\
\hline SBR grade & & & 0.091 & & & \\
\hline 1 & $1(3.3 \%)$ & $29(96.7 \%)$ & & & & \\
\hline 2 & $13(8.0 \%)$ & $150(92.0 \%)$ & & & & \\
\hline 3 & $23(12.0 \%)$ & $168(88.0 \%)$ & & & & \\
\hline Unknown & $1(2.1 \%)$ & $47(97.9 \%)$ & & & & \\
\hline Histologic type & & & 0.127 & & & \\
\hline Invasive ductal carcinoma & $36(8.5 \%)$ & $389(91.5 \%)$ & & & & \\
\hline Invasive lobular carcinoma & $1(25.0 \%)$ & $3(75.0 \%)$ & & & & \\
\hline Mucinous carcinoma & 0 & $1(100.0 \%)$ & & & & \\
\hline Others & $1(50.0 \%)$ & $1(50.0 \%)$ & & & & \\
\hline Margin & & & $>0.999$ & & & \\
\hline Free & $36(8.8)$ & $371(91.2 \%)$ & & & & \\
\hline Positive & $2(8.0 \%)$ & $23(92.0 \%)$ & & & & \\
\hline ER & & & 0.005 & & & \\
\hline Positive & $14(5.6 \%)$ & $238(94.4 \%)$ & & & & \\
\hline Negative & $24(13.3 \%)$ & $156(86.7 \%)$ & & & & \\
\hline$P R$ & & & 0.004 & & & \\
\hline Positive & $9(4.5 \%)$ & $190(95.5 \%)$ & & & & \\
\hline Negative & $29(12.4 \%)$ & $204(87.6 \%)$ & & & & \\
\hline HER2 & & & 0.151 & & & \\
\hline Positive & $21(11.0 \%)$ & $170(89.0 \%)$ & & & & \\
\hline Negative & $17(7.1 \%)$ & $224(92.9 \%)$ & & & & \\
\hline Subtype & & & 0.029 & & & \\
\hline HR +/HER2- & $7(4.6 \%)$ & $146(95.4 \%)$ & & 1 & & \\
\hline $\mathrm{HR}+/ \mathrm{HER} 2+$ & $8(7.5 \%)$ & $99(92.5 \%)$ & & 1.685 & $0.592-4.797$ & 0.328 \\
\hline HR-/HER2 + & $13(15.5 \%)$ & $71(84.5 \%)$ & & 3.819 & $1.460-9.990$ & 0.006 \\
\hline HR-/HER2- & $10(11.4 \%)$ & $78(88.6 \%)$ & & 2.674 & $0.980-7.300$ & 0.055 \\
\hline Neoadjuvant regimens & & & 0.345 & & & \\
\hline Anthracycline only & $2(18.2 \%)$ & $9(81.8 \%)$ & & & & \\
\hline Taxane only & $1(2.6 \%)$ & $37(97.4 \%)$ & & & & \\
\hline Anthracycline + taxane & $19(8.5 \%)$ & $204(91.5 \%)$ & & & & \\
\hline Chemotherapy + target therapy & $16(10.0 \%)$ & $144(90.0 \%)$ & & & & \\
\hline $\mathrm{pCR}$ & & & 0.111 & & & \\
\hline Yes & $8(5.7 \%)$ & $133(94.3 \%)$ & & & & \\
\hline No & $30(10.3 \%)$ & $261(89.7 \%)$ & & & & \\
\hline
\end{tabular}

$E R$ estrogen receptor; $H R$ hormone receptor; $H E R-2$ human epidermal growth factor receptor 2; $L R R$ locoregional recurrence; $N A C$ neoadjuvant chemotherapy; $P C R$ pathological complete response; $P R$ progesterone receptor 


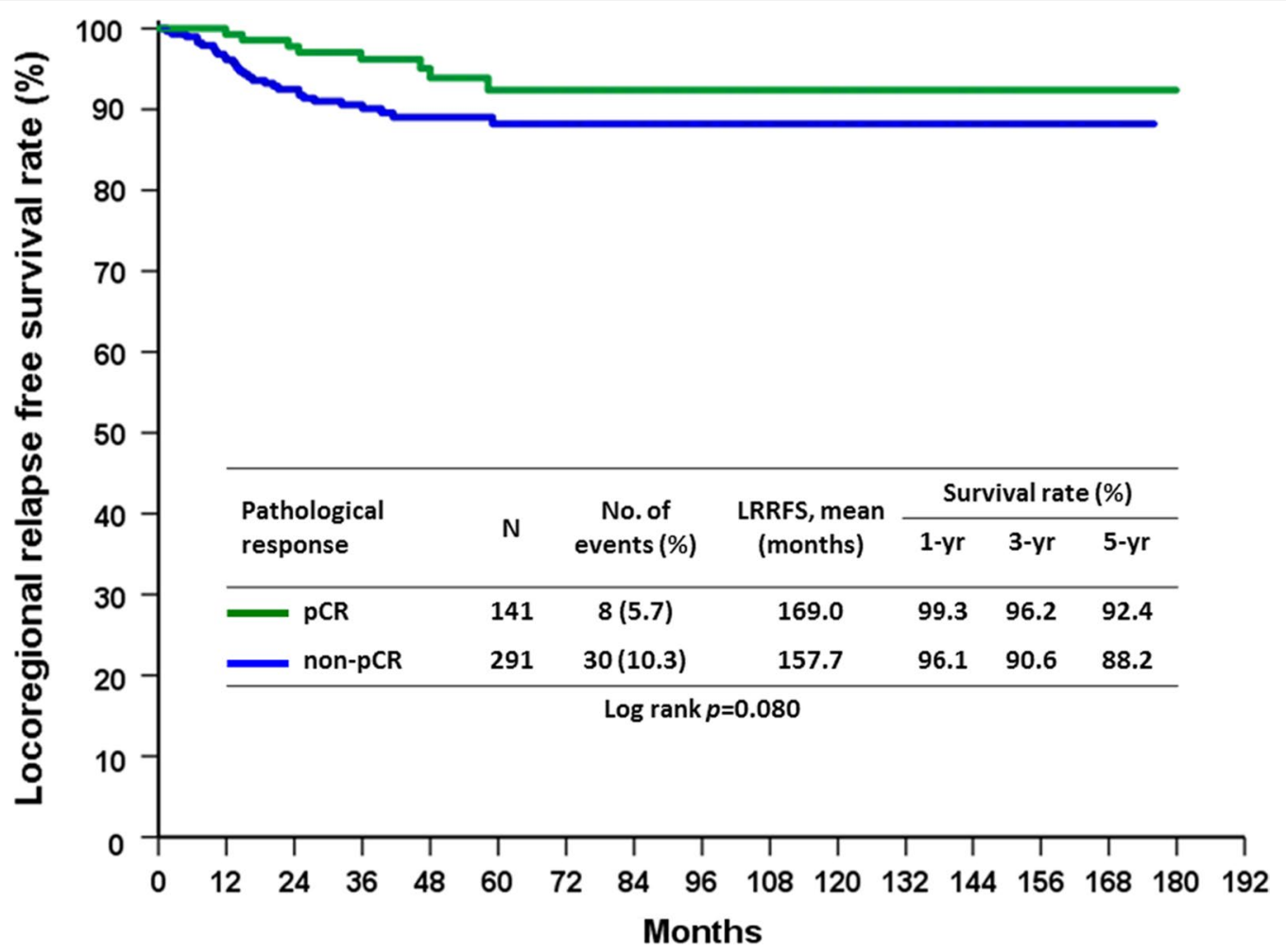

Fig. 4 LRRFS rate in the $p C R$ group compared with the non-pCR group in patients who underwent BCS

Table 8 Comparison of LRR in pCR and non-pCR patients after BCS and mastectomy in different subtypes

\begin{tabular}{|c|c|c|c|c|c|}
\hline \multirow{3}{*}{$\begin{array}{l}\text { Parameters } \\
\text { Operation type }\end{array}$} & \multirow[t]{3}{*}{ Subtype } & \multirow[t]{3}{*}{ Recurrence patterns } & \multicolumn{2}{|c|}{ Pathological total response } & \multirow{3}{*}{$P$ value } \\
\hline & & & $\mathrm{pCR}$ & Non-pCR & \\
\hline & & & $(N=91)$ & $(N=524)$ & \\
\hline \multirow{8}{*}{$\begin{array}{l}\text { Mastectomy } \\
(\mathrm{N}=615)\end{array}$} & $\mathrm{HR}+/ \mathrm{HER} 2-$ & LRR & $1(9.1 \%)$ & $25(9.8 \%)$ & $>0.999$ \\
\hline & $(N=266)$ & No LRR & $10(90.9 \%)$ & $230(90.2 \%)$ & \\
\hline & $\mathrm{HR}+/ \mathrm{HER} 2+$ & LRR & 0 & $11(10.8 \%)$ & 0.12 \\
\hline & $(N=130)$ & No LRR & $28(100 \%)$ & $91(89.2 \%)$ & \\
\hline & HR-/HER2 + & LRR & $1(2.5 \%)$ & $15(14.3 \%)$ & 0.071 \\
\hline & $(N=145)$ & No LRR & $39(97.5 \%)$ & $90(85.7 \%)$ & \\
\hline & HR-/HER2- & LRR & $2(16.7 \%)$ & $11(17.7 \%)$ & $>0.999$ \\
\hline & $(N=74)$ & No LRR & $10(83.3 \%)$ & $51(82.3 \%)$ & \\
\hline \multirow{8}{*}{$\begin{array}{l}\text { Conserving breast surgery } \\
(n=432)\end{array}$} & $\mathrm{HR}+/ \mathrm{HER} 2-$ & LRR & $1(6.3 \%)$ & $6(4.4 \%)$ & 0.546 \\
\hline & $(N=153)$ & No LRR & $15(93.7 \%)$ & $131(95.6 \%)$ & \\
\hline & $\mathrm{HR}+/ \mathrm{HER} 2+$ & LRR & $3(7.9 \%)$ & $5(7.2 \%)$ & $>0.999$ \\
\hline & $(N=107)$ & No LRR & $35(92.1 \%)$ & $64(92.8 \%)$ & \\
\hline & $\mathrm{HR}-/ \mathrm{HER} 2+$ & LRR & $4(8.3 \%)$ & $9(25.0 \%)$ & 0.037 \\
\hline & $(\mathrm{N}=84)$ & No LRR & $44(91.7 \%)$ & $27(75.0 \%)$ & \\
\hline & HR-/HER2- & LRR & 0 & $10(20.4 \%)$ & 0.002 \\
\hline & $(\mathrm{N}=88)$ & No LRR & $39(100 \%)$ & $39(79.6 \%)$ & \\
\hline
\end{tabular}


(41.3\%) received BCS. However, the choice of surgery types did not affect the LRR rate-10.7\% in mastectomy patients and $8.8 \%$ in BCS patients in the total population.

Subgroup analysis revealed 38 cases of LRR (8.8\%) following BCS, in which $5.7 \%$ in pCR group and $10.3 \%$ in non-pCR group. Further investigation according to the molecular subtype showed that in the BCS group, HR-I HER2 + non-pCR patients had significantly increased LRR than $\mathrm{HR}-/ \mathrm{HER} 2+\mathrm{pCR}$ patients and that HR-/ HER2 - non-pCR patients had a significantly increased LRR than HR-/HER2-pCR patients. In the mastectomy group, an increasing trend with regards to the risk of LRR in the non-pCR group was observed, but this was not significant. Caudle et al. reported that HR-/HER2 + and HR-/HER2- patients with a poor response to NAC had worse LRRFS after BCS. Furthermore, the authors found that patients with HR + /HER2 - and HR + /HER2 + subtypes had excellent LRRFS, regardless of tumor response to NAC[1]. Another study group from Korea revealed HR-/HER2- subtypes and HER2 + without trastuzumab subtypes predicted higher rates of LRR after NAC and $\mathrm{BCT}$, while A pCR was predictive of improved LRR in HR-/HER2 - subtype [2]. Moreover, the I-SPY 1 Trial reported that the 5 -year local recurrence risk was $0 \%$ for mastectomy and $9 \%$ for breast conservation in patients with an excellent response to NAC while the local recurrence rate was $12 \%$ for mastectomy and $7 \%$ for breast conservation in significant residual disease [21]. Another critical point was the resection area of operation after NAC. In patients who undergo NAC in order to achieve breast-tumor downstaging to enable BCS, the tumor site should be marked with a clip before initiating NAC, and resection of the entire volume of breast tissue originally occupied by tumor is not necessary [25]. However, the difference of resection area influencing local recurrence was still unknown in neoadjuvant setting. Local control appeared to be worse in HR- subtype non-pCR BCS patients after NAC in our study, but the effect on overall survival remains unknown. Further investigation is needed to determine overall survival outcomes.

\section{Limitation}

One of the limitations of this study is that it was a retrospective study from a single institution, which may result in selection bias. This study is also limited by the relatively short follow-up period. Furthermore, not all patients with the same subtype diagnosis received the same chemotherapy regimens.

\section{Conclusion}

Clinical N2 status, negative ER, and failure to achieve pCR after NAC were independently related to the risk of developing LRR. Operation type did not impact on the
LRR. In addition, the LRR rate was higher in non-pCR hormone receptor-negative patients undergoing BCS comparing with $\mathrm{pCR}$ patients. Some strategies to guide adjuvant treatment and strict follow up are needed in non-pCR hormone receptor-negative patients undergoing BCS after NAC.

\section{Abbreviations \\ NAC: Neoadjuvant chemotherapy; BCS: Breast conservation surgery; LRR: Locoregional recurrence; LRRFS: Locoregional recurrence free survival; CR: Pathological complete response; HR: Hormone receptor; HER2: Human epider- mal growth factor receptor.}

\section{Acknowledgements}

Not applicable.

\section{Authors' contributions}

HC, WC and RD analyzed and interpreted the patient data, and were major contributors in writing the manuscript and performed the analysis of statistics; YL and SC supervised the manuscript. Other authors collected the clinical data. All authors read and approved the final manuscript.

\section{Funding}

This study was supported by research plan in Chang Gung Memorial Hospital, study number CMRPG1 K0061, including the support of data collection and the statisticians.

\section{Availability of data and materials}

The datasets used and/or analysed during the current study are available from the corresponding author on reasonable request.

\section{Declarations}

Ethics approval and consent to participate

This study was approved by ethics committee of our institution and institutional review board (IRB) number was 1711150042 . The requirement for patient informed consent was waived according to the IRB by Chang Gung Medical Foundation.

\section{Consent for publication}

Not applicable.

\section{Competing interests}

The authors declare that they have no competing interests.

\section{Author details}

${ }^{1}$ Department of General Surgery, Chang Gung Memorial Hospital, Linkou, No. 5, Fuxing St., Guishan Dist., Taoyuan City 333 , Taiwan. ${ }^{2}$ Graduate Institute of Clinical Medical Sciences, College of Medicine, Chang Gung University, No. 259, Wenhua 1st Rd., Guishan Dist., Taoyuan City 333, Taiwan. ${ }^{3}$ Colleges of Medicine, Chang Gung University, No. 259, Wenhua 1st Rd., Guishan Dist., Taoyuan City 333, Taiwan.

Received: 6 July 2020 Accepted: 15 March 2021

Published online: 23 March 2021

References

1. Caudle AS, Yu TK, Tucker S, Bedrosian I, Litton JK, Gonzalez-Angulo AM, et al. Local-regional control according to surrogate markers of breast cancer subtypes and response to neoadjuvant chemotherapy in breast cancer patients undergoing breast conserving therapy. Breast Cancer Res. 2012;14(3):R83.

2. Jwa E, Shin KH, Kim JY, Park YH, Jung SY, Lee ES, et al. Locoregional recurrence by tumor biology in breast cancer patients after preoperative 
chemotherapy and breast conservation treatment. Cancer Res Treat. 2016;48(4):1363-72.

3. Veiga DF, Campos FSM, Ribeiro LM, Archangelo I Jr, Veiga Filho J, Juliano Y, et al. Mastectomy versus conservative surgical treatment: the impact on the quality of life of women with breast cancer. Revista Brasileira Saúde Materno Infantil. 2010;10:1-57.

4. Asselain B, Barlow W, Bartlett J, Early Breast Cancer Trialists' Collaborative G. Long-term outcomes for neoadjuvant versus adjuvant chemotherapy in early breast cancer: meta-analysis of individual patient data from ten randomised trials. Lancet Oncol. 2018;9(1):27-39.

5. Mauri D, Pavlidis N, loannidis JPA. Neoadjuvant versus adjuvant systemic treatment in breast cancer: a meta-analysis. JNCI. 2005;97(3):188-94.

6. van Dongen JA, Voogd AC, Fentiman IS, Legrand C, Sylvester RJ, Tong D, et al. Long-term results of a randomized trial comparing breast-conserving therapy with mastectomy: European Organization for Research and Treatment of Cancer 10801 Trial. JNCI. 2000;92(14):1143-50.

7. Veronesi U, Cascinelli N, Mariani L, Greco M, Saccozzi R, Luini A, et al. Twenty-year follow-up of a randomized study comparing breast-conserving surgery with radical mastectomy for early breast cancer. NEJM. 2002;347(16):1227-32.

8. Sun Y, Liao MJ, He L, Zhu CF. Comparison of breast-conserving surgery with mastectomy in locally advanced breast cancer after good response to neoadjuvant chemotherapy: a PRISMA-compliant systematic review and meta-analysis. Medicine (Baltimore). 2017;96(43):8367.

9. Zhou X, Li Y. Local recurrence after breast-conserving surgery and mastectomy following neoadjuvant chemotherapy for locally advanced breast cancer-a meta-analysis. Breast Care (Basel). 2016;1 1(5):345-51.

10. Mamounas EP, Anderson SJ, Bear HD, Julian TB, Geyer CE Jr, Taghian A, et al. Predictors of locoregional recurrence after neoadjuvant chemotherapy: results from combined analysis of National Surgical Adjuvant Breast and Bowel Project B-18 and B-27. J Clin Oncol. 2012;30(32):3960-6.

11. Matsuda N, Hayashi N, Ohde S, Yagata H, Kajiura Y, Yoshida A, et al. A nomogram for predicting locoregional recurrence in primary breast cancer patients who received breast-conserving surgery after neoadjuvant chemotherapy. J Surg Oncol. 2014;109(8):764-9.

12. Chou HH, Kuo WL, Yu CC, Tsai HP, Shen SC, Chu CH, et al. Impact of age on pathological complete response and locoregional recurrence in locally advanced breast cancer after neoadjuvant chemotherapy. Biomed J. 2019;42:66-74.

13. Cortazar P, Zhang L, Untch M, Mehta K, Costantino JP, Wolmark N, et al. Pathological complete response and long-term clinical benefit in breast cancer: the CTNeoBC pooled analysis. Lancet. 2014;384:164-72.

14. von Minckwitz G, Untch M, Blohmer JU, Costa SD, Eidtmann H, Fasching PA. Definition and impact of pathologic complete response on prognosis after neoadjuvant chemotherapy in various intrinsic breast cancer subtypes. J Clin Oncol. 2012;30:1796-804.

15. Spring LM, Fell G, Afre A, Sharma C, Greenup RA, Reynolds KL, et al. Pathological complete response after neoadjuvant chemotherapy and impact on breast cancer recurrence and survival: a comprehensive meta-analysis. Clin Cancer Res. 2020;26:2838-48.

16. Gillon P, Touati N, Breton-Callu C, Slaets L, Cameron D, Bonnefoi H. Factors predictive of locoregional recurrence following neoadjuvant chemotherapy in patients with large operable or locally advanced breast cancer: an analysis of the EORTC 10994/BIG 1-00 study. Eur J Cancer. 2017:79:226-34.

17. Swisher SK, Vila J, Tucker SL, Bedrosian I, Shaitelman SF, Litton JK, et al. Locoregional control according to breast cancer subtype and response to neoadjuvant chemotherapy in breast cancer patients undergoing breast-conserving therapy. Ann Surg Oncol. 2016;23:749-56.

18. Yang TJ, Morrow M, Modi S, Zhang Z, Krause K, Siu C, et al. The effect of molecular subtype and residual disease on locoregional recurrence in breast cancer patients treated with neoadjuvant chemotherapy and postmastectomy radiation. Ann Surg Oncol. 2015;22:495-501.

19. Bear HD, Anderson S, Smith RE, Geyer CE Jr, Mamounas EP, Fisher B, et al. Sequential preoperative or postoperative docetaxel added to preoperative doxorubicin plus cyclophosphamide for operable breast cancer: National Surgical Adjuvant Breast and Bowel Project protocol B-27. J Clin Oncol. 2006;24:2019-27.

20. Untch M, Fasching PA, Konecny GE, Hasmüller S, Lebeau A, Kreienberg $R$, et al. Pathologic complete response after neoadjuvant chemotherapy plus trastuzumab predicts favorable survival in human epidermal growth factor receptor 2-overexpressing breast cancer: results from the TECHNO trial of the AGO and GBG study groups. J Clin Oncol. 2011;29:3351-7.

21. Cureton EL, Yau C, Alvarado MD, Krontiras H, Ollila DW, Ewing CA, et al. Local recurrence rates are low in high-risk neoadjuvant breast cancer in the I-SPY 1 Trial (CALGB 150007/150012; ACRIN 6657). Ann Surg Oncol. 2014;21:2889-96.

22. Buchholz TA, Mittendorf EA, Hunt KK. Surgical considerations after neoadjuvant chemotherapy: breast conservation therapy. J Natl Cancer Inst Monogr. 2015;2015:11-4.

23. van der Hage JA, van de Velde CJ, Julien JP, Tubiana-Hulin M, Vandervelden C, Duchateau L. Preoperative chemotherapy in primary operable breast cancer: results from the European Organization for Research and Treatment of Cancer trial 10902. J Clin Oncol. 2001;19(22):4224-37.

24. Mieog JS, van der Hage JA, van de Velde CJ. Neoadjuvant chemotherapy for operable breast cancer. Br J Surg. 2007;94:1 189-200.

25. Boughey JC, Peintinger F, Meric-Bernstam F, Perry AC, Hunt KK, Babiera GV, et al. Impact of preoperative versus postoperative chemotherapy on the extent and number of surgical procedures in patients treated in randomized clinical trials for breast cancer. Ann Surg. 2006;244:464-70.

\section{Publisher's Note}

Springer Nature remains neutral with regard to jurisdictional claims in published maps and institutional affiliations.

Ready to submit your research? Choose BMC and benefit from

- fast, convenient online submission

- thorough peer review by experienced researchers in your field

- rapid publication on acceptance

- support for research data, including large and complex data types

- gold Open Access which fosters wider collaboration and increased citations

- maximum visibility for your research: over $100 \mathrm{M}$ website views per year

At BMC, research is always in progress.

Learn more biomedcentral.com/submissions 